\title{
RPA-PCR couple: an approach to expedite plant diagnostics and overcome PCR inhibitors
}

Mustafa Ahmad Munawar*, 1 iD, Frank Martin², Anna Toljamo', Harri Kokko' \& Elina Oksanen'

${ }^{1}$ Department of Environmental \& Biological Sciences, University of Eastern Finland, Kuopio 70211, Finland; ${ }^{2}$ US Department of Agriculture, ARS, Salinas, CA 93905, USA;

*Author for correspondence: mustafm@student.uef.fi

BioTechniques 69: 271-280 (October 2020) 10.2144/btn-2020-0065

First draft submitted: 6 May 2020; Accepted for publication: 10 July 2020; Published online: 20 August 2020

\section{ABSTRACT}

DNA extraction can be lengthy and sometimes ends up with amplification inhibitors. We present the potential of recombinase polymerase amplification (RPA) to replace plant DNA extraction. In our rapid 'RPA-PCR couple' concept, RPA is tuned to slower reaction kinetics to promote amplification of long targets. RPA primers amplify target and some flanking regions directly from simple plant macerates. Then PCR primers exponentially amplify the target directly from the RPA reaction. We present the coupling of RPA with conventional, TaqMan and SYBR Green PCR assays. We applied the concept to strawberry Phytophthora pathogens and the Phytophthora identification marker atp9-nad9. We found RPA-PCR couple specific, sensitive and reliable. The approach may also benefit other difficult samples such as food, feces and ancient samples.

\section{METHOD SUMMARY}

Crudely macerate plant tissue in water for a few minutes and transfer supernatant in a recombinase polymerase amplification (RPA) reaction. Incubate RPA reaction for $20 \mathrm{~min}$ at $39^{\circ} \mathrm{C}$ and thereafter directly utilize RPA reaction to exponentially amplify target through standard PCR.

\section{GRAPHICAL ABSTRACT}

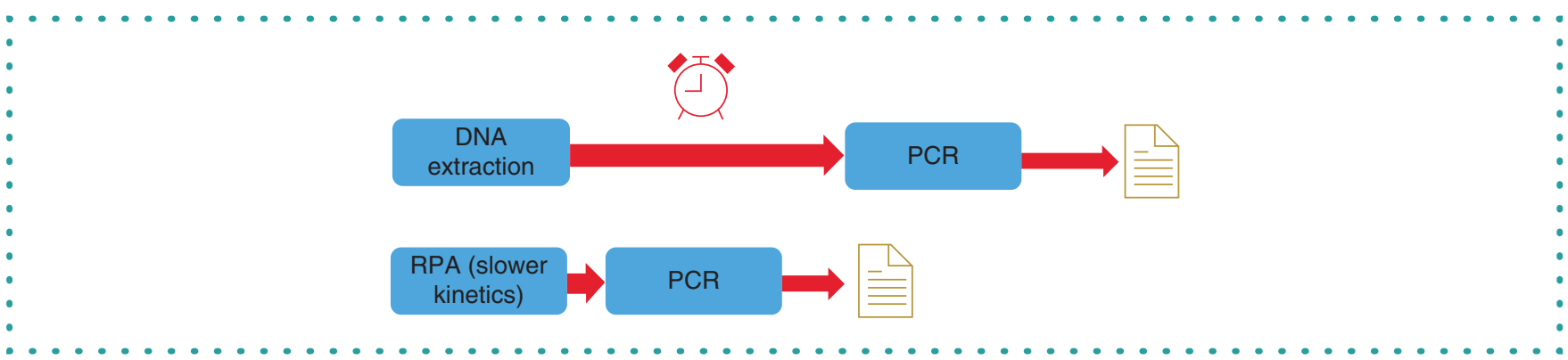

\section{KEYWORDS:}

atp9-nad9 • PCR inhibitors • Phytophthora • Phytophthora cactorum • Phytophthora fragariae • plant DNA extraction • recombinase polymerase amplification

A standard method for diagnosis of plant diseases is PCR. Traditionally, PCR requires prior extraction of DNA from infected tissue. Plant DNA extraction not only is a time-consuming process but also sometimes co-extracts PCR inhibitors. To identify the presence of inhibitors in DNA samples, amplification of internal controls are often measured to ensure amplification efficiency is not affected. Although the internal controls spot false negatives, they also can increase cost and reduce amplification of the target amplicon when it is present in low concentrations. To combat reductions in amplification efficiency due to inhibitors, DNA extracts can be further purified, diluted or have the DNA extraction repeated. Although DNA dilution can reduce concentration of inhibitors, it also impacts the sensitivity of the assay by reducing the number of target DNA copies. For these reasons, DNA extraction from infected plant material may be a bottleneck in diagnosis of diseases [1].

An alternative DNA amplification method - recombinase polymerase amplification (RPA) - has been developed that addresses many of these limitations of DNA extraction [2]. RPA is an isothermal amplification where primers of 30-35 nucleotides length bind with recombinase enzyme, and these nucleoprotein complexes scan DNA to pair up with a homologous region. This results in D-loop structure formation, where recombinase dissociates from primers and polymerase extend the primers. Bidirectional extension of primers by polymerase with strand displacement activity leads to exponential amplification of the target nucleic acid. Commercially available formulations for RPA assays can be obtained from TwistDx Inc. (Maidenhead, UK) the patent holder of the technology, and Agdia (Soisy-sur-Seine, 
France). TwistDx Inc., in addition to supplying reagents for RPA technology, aims at supporting the deployment of RPA technology in various fields including life sciences, diagnostics, microfluidics/lab-on-chip applications, agriculture and defense.

RPA is reported to be tolerant of PCR inhibitors [3] and can amplify DNA directly from crudely macerated plant tissue. Crude macerates have been prepared with different buffers. Ready-to-use buffers from Agdia such as GEB [4], GEB 2 [5,6], GEB 4 [7] and AMP1 [8,9] have been utilized. Similarly, $\mathrm{NaOH}$ [10,11], standard ELISA grinding buffer [5] and TE buffer [12] have also been used for maceration. Besides maceration, extraction buffers have been employed to release nucleic acid from leaf disks [13,14]. Moreover, squeezed sap has been directly utilized for RPA [15].

In addition to tolerances to inhibitors, RPA is rapid and sensitive, and it requires simple instrumentation. For these reasons, RPA has gained attention from plant pathologists, but users may encounter some challenges when designing amplification primers with the necessary specificity. Although many researchers have reported satisfactory specificity of their RPA assays, some have also clued about the nonspecificity issue [16-18]. RPA assays have also been reported tolerating five mismatches [19], seven mismatches [20] and even nine mismatches [21] with target sites. The study reporting tolerance up to seven mismatches originally investigated the effect of different mismatches on RPA specificity and recommended selection of a target marker with at least $36 \%$ sequence difference with the closely related species while designing RPA assays [20]. RPA primers, in contrast to PCR, do not depend on melting temperatures to anneal with target sites. Amplification at low temperature ranging from 25 to $42^{\circ} \mathrm{C}$ may enable RPA primers to make dimers and even bind to matching nontarget sites, thereby generating nonspecific by-products that can be visible on an agarose gel. Moreover, a nonspecific byproduct longer than 300 base pairs (bp) cannot be removed through a routine PCR purification kit, and in such a scenario, gel extraction of the target amplicon would be necessary. In an RPA reaction, the nonspecific products also compete with the target amplification for the limited energy available, and therefore targets are kept short, ideally of 100-200 bp. Longer amplicons require lengthier extension time, and thereby target amplification may be outcompeted by the background or nonspecific amplification. For longer amplicons, TwistDx manuals recommend slower reaction kinetics through lowering the incubation temperature and concentration of magnesium acetate and primers. In our experience, lowering the concentration of magnesium acetate and primers is beneficial for longer amplicons, but detection of a low amount of longer targeted amplicons ( $500 \mathrm{bp})$ is still not possible. It should be noted that background amplification is less problematic for the real-time RPA chemistries designed for inclusion of a probe-based detection system, such as the TwistAmp exo kit.

Although both PCR and RPA have their disadvantages, a unique combination of them can result in improved detection capabilities. Coupling slower reaction kinetics RPA with PCR can exclude the need for time-consuming and problematic DNA extractions. The method is also expected to overcome the RPA shortcomings of nonspecificity (background amplification), short amplicon size (100-200 bp optimal) and poor resolution for quantification. In the coupled amplification, RPA slowly multiplies the target and some flanking region in the first-round amplification directly from plant macerate. Then in the second round of amplification, PCR exponentially amplifies the target region directly from the RPA reaction. Slower RPA reaction kinetics promote generation of longer amplicons. Similarly, the slower rate of RPA amplification generates less background noise and target amplicons that can be directly utilized for PCR (without dilution). The coupled nested PCR are run with normal chemistry.

In this proof-of-concept study, we will validate RPA-PCR couple as a simple, rapid, sensitive and specific approach to directly amplify target DNA from plant crude macerates. The study will target Phytophthora intergenic mitochondrial marker atp9-nad9, and the concept will be applied to two Phytophthora species causing devastating damage to strawberry. The atp9-nad9 marker is a gene order that is highly conserved in the genus Phytophthora but different in the related genus Pythium, Eumycotan fungi and plants [5,22]. The pathogens will include Phytophthora fragariae, a causative agent of red stele of strawberry roots, and Phytophthora cactorum, an agent of strawberry crown rot. Our first round of RPA assay will be Phytophthora genus-specific, and it will amplify the intergenic spacer between the atp 9 and nad9 genes at slower reaction kinetics. Then the RPA reaction will be coupled either with species-specific real-time PCR assays or genus-specific conventional PCR aimed at Sanger sequencing. The species-specific assays include SYBR Green and TaqMan assays for $P$. fragariae and $P$. cactorum, and their specificity originates from the intergenic spacer between atp 9 and nad 9 .

\section{Materials \& methods}

RPA primers screening

Supplemental file 2 of Miles et al. [5] provided 274 partial atp9-nad9 sequences of approximately 100 Phytophthora species. The sequences were aligned by Geneious 8.1.9 (Geneious, Auckland, New Zealand) to identify the atp 9 gene, intergenic spacer and nad 9 gene sequences. Ten forward RPA primers were picked from the atp 9 region and ten reverse primers from the nad 9 region. Primers were screened to find the optimal RPA primer pair generating the maximum amount of target amplicons. DNA extracted from $P$. fragariae hyphae was utilized as template, and the DNA volume was kept at $1 \mu$ per reaction. DNA extraction from Phytophthora hyphae was described previously [23]. The concentration of the hyphal DNA was $100 \mathrm{pg} / \mu \mathrm{l}$. All DNA concentrations in this study were quantified through Qubit 2.0 Fluorometer, utilizing the Qubit dsDNA HS Assay kit (Thermo Scientific, USA).

In primers screening, RPA reactions were run at normal reaction kinetics utilizing the TwistAmp Liquid Basic kit (TwistDx Inc.) and following manufacturer recommendations. RPA incubation was done at $39^{\circ} \mathrm{C}$ for 25 min with no agitation and using a PTC-0200 DNA Engine Cycler (BIO-RAD, USA) with no lid heating. Following incubation, reactions were purified with a GeneJET PCR Purification kit 


\begin{tabular}{|c|c|c|}
\hline Primer/probe & Sequence & Assay and specificity \\
\hline ATP9F-M9 & ССTTCTTTACAACAAGAATTAATGAGAAC & $\begin{array}{l}\text { RPA forward primer, genus specific (Miles et al. [5] forward primer Atp9-F with } \\
6 \text { bp truncated from the } 3^{\prime} \text { end) }\end{array}$ \\
\hline Phy_Gen_R20 & CGAATACTTAATAAATTATAAATAATTTCA & RPA reverse primer, genus specific \\
\hline Phy_Gen_SeqF4 & ACAACAAGAATTAATGAGAACTGC & $\begin{array}{l}\text { Forward primer in all PCRs, genus specific (the first } 16 \mathrm{bp} \text { are from the } 3^{\prime} \text { end of } \\
\text { PhyG_ATP9_2FTail of Bilodeau et al. [22]) }\end{array}$ \\
\hline Phy_Frag_PCRR4 & TTTTTGTTTGAAAAGAGCTA & $\begin{array}{l}\text { Reverse PCR primer in Phytophthora fragariae SYBR Green PCR assay, species } \\
\text { specific }\end{array}$ \\
\hline Phy_Cac_PCRR2 & AATAGTGTTAATAATTATATAACATG & $\begin{array}{l}\text { Reverse PCR primer in Phytophthora cactorum SYBR Green PCR assay, species } \\
\text { specific (designed from the P. cactorum TaqMan probe described in Bilodeau } \\
\text { et al. [22]) }\end{array}$ \\
\hline Phy_Gen_SeqR8 & GGTAAAATTTGTAATAAATATTGACT & Reverse PCR primer in conventional and TaqMan PCRs, genus specific \\
\hline PfraVf_nad9sp_TaqMan2 & $\begin{array}{l}\text { /56-FAM/ATC TCG TAA /ZEN/TAG ATA TAT ATG TAT } \\
\text { ATT TAA TAC GT/3IABkFQ/ }\end{array}$ & Probe in $P$. fragariae TaqMan PCR assay, species specific [22] \\
\hline Pcact_nad9sp_probe2 & $\begin{array}{l}\text { /56-FAM/TTA CAT GTT /ZEN/ATA TAA TTA TTA } \\
\text { ACA CTA TTT ATA AAA /3IABkFQ/ }\end{array}$ & Probe in P. cactorum TaqMan PCR assay, species specific [22] \\
\hline
\end{tabular}

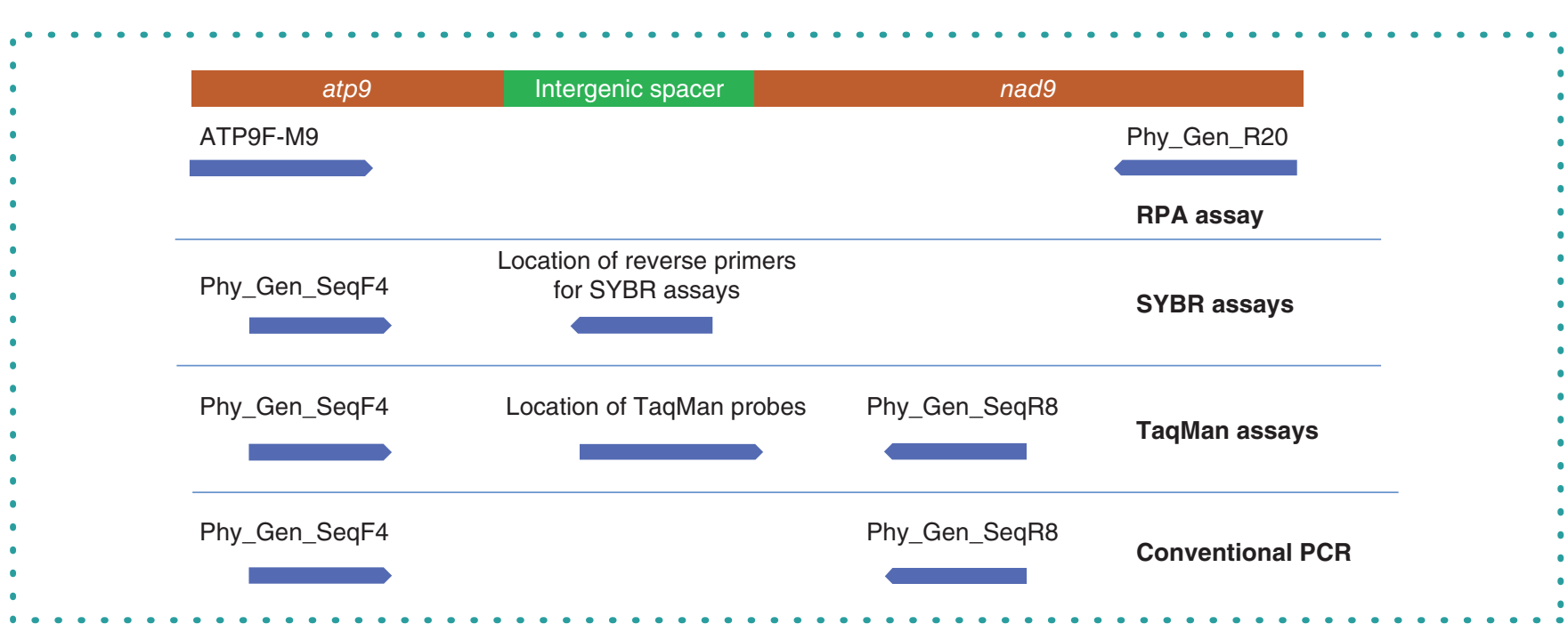

Figure 1. Location of primers in recombinase polymerase amplification, SYBR Green PCR, TaqMan PCR and conventional PCR assay.

(Thermo Scientific) and results were examined by the end point determination method of gel electrophoresis. The forward RPA primer 'ATP9F-M9' and reverse RPA primer 'Phy_Gen_R20' (Table 1) were found to be the optimum RPA primer pair (data not shown).

\section{Real-time SYBR Green PCR assays}

All PCR reactions in this study were performed with 500-nM primer concentration and an amplification volume of $25 \mu$ l. PCR primers were designed through Geneious 8.1.9, where default GC\% and temperature of melting (Tm) were often lowered. All quantitative PCR amplifications were run with the Mx3000P QPCR System (Agilent, Waldbronn, Germany). All primers and probes were ordered from Integrated DNA Technologies Inc. (Leuven, Belgium). Primers and probe sequences are provided in Table 1, with their locations in the atp9-nad9 region presented in Figure 1.

In species-specific SYBR Green PCR assays, reverse primers were designed from the intergenic spacer between the atp 9 and nad9 genes, and forward primers from the genus conserved region of atp9. In the $P$. fragariae assay, the forward primer 'Phy_Gen_SeqF4' and reverse primer 'Phy_Frag_PCRR4' were utilized. The amplification program included 10-min predenaturation at $95^{\circ} \mathrm{C}$, and 40 amplification cycles, where each cycle has 10 -s denaturation at $95^{\circ} \mathrm{C}, 20$-s annealing at $58^{\circ} \mathrm{C}$ and extension of $20 \mathrm{~s}$ at $67^{\circ} \mathrm{C}$. The $P$. cactorum assay was done with the forward primer 'Phy_Gen_SeqF4' and reverse primer 'Phy_Cac_PCRR2.' Amplification conditions were similar to $P$. fragariae with the exception of a $55^{\circ} \mathrm{C}$ annealing temperature and extension of $30 \mathrm{~s}$ at $60^{\circ} \mathrm{C}$. All SYBR Green assays were completed with LightCycler ${ }^{\circledR} 480$ SYBR Green I Master (Roche, Rotkreuz, Switzerland). Melt curve analysis was always included in SYBR Green amplifications. 
Real-time TaqMan assays

Bilodeau et al. [22] designed and validated species-specific TaqMan assays for several Phytophthora species including those used in this experimentation. In these assays, probes were placed in the intergenic spacer between the atp 9 and nad 9 genes, whereas forward and reverse primers were designed from the flanking genus conserved regions. The probes for $P$. fragariae 'PfraVf_nad9sp_TaqMan2' and for $P$. cactorum 'Pcact_nad9sp_probe2' were ordered as ZEN double-quenched probes to improve signal to background discrimination. The forward primer 'PhyG_ATP9_2FTail' and reverse primer 'PhyG-R6_Tail' were replaced with forward primer 'Phy_Gen_SeqF4' and reverse primer 'Phy_Gen_SeqR8,' as the latter improved sensitivities. In the species-specific TaqMan PCR assays, probe concentration was kept at $100 \mathrm{nM}$, and Luminaris Probe qPCR Master Mix (Thermo Scientific) was used. The PCR program for the $P$. fragariae assay started with a 2-min incubation at $50^{\circ} \mathrm{C}$ to let Uracil-DNA glycosylase (UDG) remove possible carryover contamination. Then a 10-min incubation at $95^{\circ} \mathrm{C}$ to activate Hot Start Taq DNA polymerase followed by 45 amplification cycles. Each cycle consisted of a denaturation step of $15 \mathrm{~s}$ at $95^{\circ} \mathrm{C}$ and an annealing/ extension step of $90 \mathrm{~s}$ at $57^{\circ} \mathrm{C}$. The amplification program for the $P$. cactorum assay was the same but with an annealing/extension step at $55^{\circ} \mathrm{C}$.

\section{Conventional PCR for sequencing}

Conventional PCR amplification of the Phytophthora genus-specific amplicon was done to generate a template for Sanger sequencing. The amplification utilized the previously mentioned 'Phy_Gen_SeqF4' and 'Phy_Gen_SeqR8' primers. LightCycler ${ }^{\circledR} 480$ SYBR Green I Master was utilized as the master mix due to its exceptional capability to amplify even after 50 cycles. The fluorescence signals were not considered; rather, gel electrophoresis was utilized to observe amplification of the target approximately $300 \mathrm{bp}$ bands. The amplification program started with a 10-min preincubation at $95^{\circ} \mathrm{C}$ and followed 50 amplification cycles of $10 \mathrm{~s}$ at $95^{\circ} \mathrm{C}, 20 \mathrm{~s}$ at $55^{\circ} \mathrm{C}$ and $30 \mathrm{~s}$ at $65^{\circ} \mathrm{C}$. PCR reactions with visible target bands were purified with GeneJET PCR Purification and sent for Sanger sequencing to GATC Biotech (Konstanz, Germany).

\section{Coupling RPA with PCR}

For the RPA-PCR couple method, the TwistAmp Liquid Basic kit was used to prepare an RPA reaction mix for amplifying a target of approximately $500 \mathrm{bp}$ under conditions of slower reaction kinetics. A premaster mix was prepared in a $1.5-\mathrm{ml}$ tube. The premaster mix $1 X$ recipe included $25 \mu \mathrm{l}$ of $2 \times$ reaction buffer, $2 \mu \mathrm{l}$ of $10 \times$ Basic E-Mix, $1 \mu \mathrm{l}$ of $40 \mathrm{mM}$ dNTPs (total), $1.5 \mu \mathrm{l}$ of forward primer 'ATP9F-M9' $(10 \mu \mathrm{M}), 1.5 \mu \mathrm{l}$ of reverse primer 'Phy_Gen_R20' $(10 \mu \mathrm{M})$ and $15.21 \mu \mathrm{l}$ of water. The premaster mix was vortexed and spun briefly, and the $20 \times$ Core Reaction Mix was added to the lid and the tube was inverted ten-times to prepare the final master mix. For $1 \mathrm{X}$ recipe of the master mix, 20x Core Reaction Mix volume was kept at $1 \mu$. The $20 \times$ Core Reaction Mix was also warmed to room temperature and pipette mixed before use. Then $47.21 \mu$ of the master mix was added to individual amplification tubes followed by $1 \mu \mathrm{l}$ of macerate/template and $1.79 \mu \mathrm{l}$ of $280 \mathrm{mM} \mathrm{MgOAc}$ that were added to the inside of the lid strip. Reactions were started by inverting the tubes to mix and the amplification mixture concentrated at the bottom of the tube prior to immediately incubating the tubes at $39^{\circ} \mathrm{C}$ for 20 min, with an agitation step after 4 min. Following 20-min incubation, reaction enzymes were denatured by heating at $85^{\circ} \mathrm{C}$ for $1 \mathrm{~min}$ to terminate amplification and cooling to $4^{\circ} \mathrm{C}$. The RPA incubation was done in a PTC-0200 DNA Engine Cycler with no lid heating. Once the RPA reaction was completed, a 1- $\mu$ l reaction was directly utilized for PCR or frozen at $-20^{\circ} \mathrm{C}$ for future use. In RPA-PCR couple, the number of PCR amplification cycles were reduced. The coupled species-specific SYBR Green and TaqMan amplifications were done with 30 thermal cycles, whereas conventional PCR genus-specific amplification was done with 40 cycles.

For estimation of sensitivity of the RPA-PCR couple method, slower reaction kinetics RPA reactions were done with $1 \mu$ of $P$. fragariae or $P$. cactorum hyphal DNA dilutions at concentrations ranging from $100 \mathrm{pg} / \mu \mathrm{l}$ to $0.1 \mathrm{fg} / \mu \mathrm{l}$ and coupled with related PCR assays. The RPA reactions with $P$. fragariae DNA dilutions were spiked with macerate of healthy rootlet tips, whereas reactions with $P$. cactorum DNA were spiked with macerate of healthy crown tissue. Macerate preparation was described previously [23] and added in amounts that would be used for assaying plant samples $(1 \mu \mathrm{l})$.

\section{Specificity}

In laboratory evaluations of specificity, we first analyzed all PCR assays individually for possible cross reactivity with DNA from nontarget taxa. Then we analyzed RPA-PCR couple specificity by adding DNA from each taxa individually for RPA amplification and coupling those RPA reactions with all formats of PCR assays. DNA extracts concentrations ranged between 50 and $100 \mathrm{pg} / \mu \mathrm{l}$, and $1 \mu \mathrm{l}$ of each DNA extract was added in individual reaction tubes. We utilized DNA extracts of $P$. fragariae, $P$. cactorum, Phytophthora taxon raspberry, Phytophthora megasperma, Phytophthora rosacearum, Phytophthora ramorum, Phytophthora plurivora, Phytophthora pini, Phytophthora cambivora, Phytophthora cinnamomi, Pythium sylvaticum, Botrytis cinerea, Colletotrichum acutatum, Mucor hiemalis, Fusarium avenaceum and Fusarium proliferatum. All five fungal species, $P$. sylvaticum, $P$. fragariae and $P$. cactorum were isolated from strawberry plants. More details of the taxa included in laboratory evaluations of specificity are provided in Supplementary Table 1 in the Supplementary data. In addition to laboratory evaluation of specificity, we customized NCBI Primer-BLAST to analyze specificity of the species-level SYBR Green PCR assays. For this purpose, we utilized the partial atp9-nad9 sequences of approximately 100 Phytophthora species provided in supplemental file 2 of Miles et al. [5] and complete atp 9 gene sequences from 28 Phytophthora species received from the US Department of Agriculture's Agricultural Research Service (CA, USA). 


\section{Field validation}

For field validation, 22 fine rootlet samples and 22 crown samples were collected from the plants investigated for red stele and crown rot disease in the summer of 2018. The plants samples were from 25 different problematic fields of the Northern Savo region of Finland. Each rootlet sample was collected from three plants from a field (pooling of finest root tips). In contrast, crown samples were not pooled; rather, each crown sample was collected from individual plants. The crown samples always included rot if it was observed. Tissue samples weighing from 25 to $100 \mathrm{mg}$ were collected in a round bottom 2-ml Eppendorf tube and macerated in autoclaved distilled water with a plastic pestle. Rootlet macerates were prepared as 1:10 (w/v) tissue weight by volume of water, whereas crowns macerates were 1:20 (w/v). To facilitate maceration, initially only $100 \mu l$ of water was added, and following maceration, more water was supplemented to achieve the final desired dilution. Once macerates were ready, they were vortexed and left for particulate settlement for approximately $1 \mathrm{~min}$. The maceration process is explicitly explained in Munawar et al. [23]. From tissue macerates, $1 \mu$ l of supernatant was transferred to RPA amplifications done with slower reaction kinetics. Once the RPA amplification was completed, RPA reactions were coupled with the four species-specific real-time PCR assays and one conventional genus-specific PCR assay. The target bands from conventional PCR were also Sanger sequenced to confirm identification of the taxa present.

To validate RPA-PCR couple results and compare them with standard diagnostic procedure of DNA extraction-PCR, the macerates prepared were frozen at $-80^{\circ} \mathrm{C}$. Later, the samples were freeze dried, further ground with plastic pestles and DNA extracted as reported by Till et al. [24], directly starting from the step fifth of lysis buffer addition. In DNA extraction, we utilized $100 \mu$ l of liquid silica stock per tube, guanidine thiocyanate as DNA binding buffer and LB2 as lysis buffer. Following DNA extraction, DNA volumes of $0.25,0.5$ and $1 \mu l$ from each sample were tested with the four species-specific and one conventional PCR amplification. The products from conventional PCR were also Sanger sequenced. All DNA samples that showed no amplification were tested for the presence of PCR inhibitors or DNA extraction failure through a separate control PCR amplification targeting plant DNA. The control PCR was a SYBR Green PCR assay targeting plant sequences that included the FMP12b and FMP13b primers of Tooley et al. [25], master mix LightCycler ${ }^{\circledR} 480$ SYBR Green I Master and $0.25-\mu \mathrm{l}$ DNA samples. The amplification program started with 10-min incubation at $95^{\circ} \mathrm{C}$ followed by 35 amplification cycles of $95^{\circ} \mathrm{C}$ for $10 \mathrm{~s}, 55^{\circ} \mathrm{C}$ for $20 \mathrm{~s}$ and $72^{\circ} \mathrm{C}$ for $20 \mathrm{~s}$.

\section{Results \& discussion}

\section{Optimum PCR assays}

Both species-specific SYBR Green assays detected as low as $1 \mathrm{fg}$ of target genomic DNA (eight replicates) with linearity down to $10 \mathrm{fg}$. The melt curves produced double peaks as predicted by UMELT [26]. Amplification efficiency of the $P$. fragariae assay was $98.76 \%$ and the $P$. cactorum assay was $94.50 \%$. Like the SYBR Green assays, both species-specific TaqMan assays amplified as low as $1 \mathrm{fg}$ of target genomic DNA (eight replicates) with linearity down to $10 \mathrm{fg}$. Amplification efficiency of the $P$. fragariae assay was $90.10 \%$ and the $P$. cactorum assay was $82.91 \%$. The conventional PCR genus-specific assay produced visible target band of approximately $300 \mathrm{bp}$ for as low as $1 \mathrm{fg}$ of Phytophthora hyphal DNA (two replicates).

Primer-BLAST predicted cross reactivity of the $P$. fragariae SYBR Green assay with only Phytophthora alni, $P$. cambivora and Phytophthora rubi. Similarly, Primer-BLAST foretold cross reactivity of the P. cactorum SYBR Green PCR assay with Phytophthora pseudotsugae, Phytophthora hedraiandra and Phytophthora idaei. In laboratory analysis of specificity, the species-specific TaqMan and SYBR Green assays showed no cross reactivity for the taxa tested with the exception of the SYBR Green PCR assay for $P$. fragariae, which cross reacted with $P$. cambivora as predicted by Primer-BLAST. Similarly, the conventional genus-specific PCR amplified all Phytophthora species and showed no cross reactivity with any of the non-Phytophthora species DNA.

Optimizing PCR assays designed in this project from the low GC atp9-nad9 marker was challenging, especially in the case of $P$. cactorum. In the $P$. cactorum SYBR Green assay, the extension temperature was lowered to $60^{\circ} \mathrm{C}$ and an additional $10 \mathrm{~s}$ were provided to assist completion of the extension step. Moreover, for the TaqMan PCR assays, the amplification curves shapes were more linear, missing exponential and plateau phases (shown later in Figures 3, 4B \& D). This probably happened due to low Tm or GC content of the TaqMan probes. In the $P$. fragariae TaqMan assay, 'PfraVf_nad9sp_TaqMan2' has a GC content of $20.0 \%$ and Tm of $56.1^{\circ} \mathrm{C}$, whereas in the $P$. cactorum TaqMan assay, 'Pcact_nad9sp_probe2' has a GC content of only $11.1 \%$ and Tm of $53.8^{\circ} \mathrm{C}$. Ideally, the TaqMan probes used in this study should be redesigned with addition of minor groove binder to raise $\mathrm{Tm}$ to an adequate level. Otherwise, the TaqMan probe should be further elongated to raise Tm. Regarding the Phytophthora genus-specific conventional PCR, the extension temperature was lowered to $65^{\circ} \mathrm{C}$. An extension temperature of $68^{\circ} \mathrm{C}$ worked for $P$. fragariae but failed for $P$. cactorum.

\section{Optimum RPA-PCR couple}

In sensitivity evaluation of RPA-PCR couple, slower reaction kinetics RPA reaction were done separately for $1 / 10$ serial dilutions of $P$. fragariae and $P$. cactorum hyphal DNA. The RPA reactions were also spiked with healthy rootlets and crown macerate, respectively. Following amplification, aliquots of the RPA reactions were transferred to real-time TaqMan and SYBR Green PCR assays. RPA-PCR couple consistently amplified target Phytophthora hyphal DNA down to 10-fg starting concentration (six replicates). Figure 2 represents standard curves when slower kinetics RPA reactions were done with $1 / 10$ serial dilution of $P$. fragariae DNA and healthy rootlets macerates, and the RPA reactions were coupled with $P$. fragariae specific SYBR Green PCR assay. Similarly, Figure 3 represents a similar setup of RPA-PCR coupled where RPA reactions were used with dilutions of $P$. cactorum DNA and spiked with healthy crown macerate, and later 


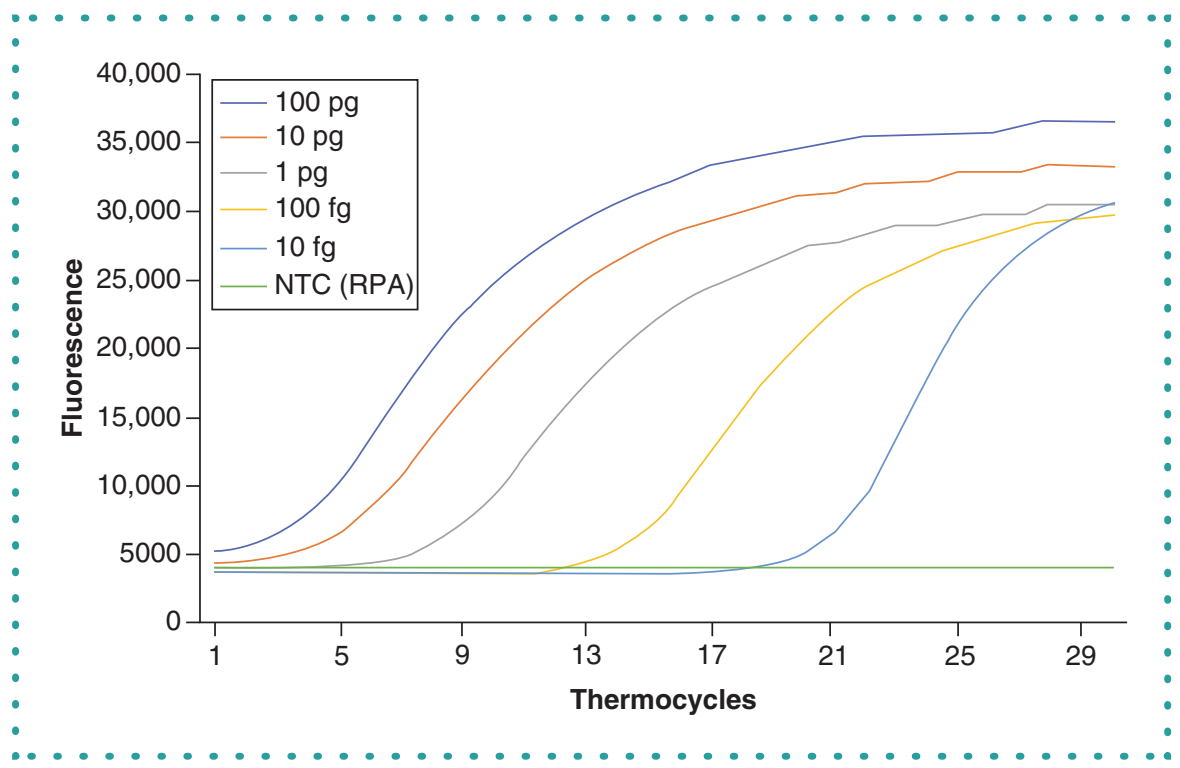

Figure 2. Standard curve of recombinase polymerase amplification-PCR couple when the template was Phytophthora fragariae DNA and the coupled PCR is a $P$. fragariae specific SYBR Green PCR assay. The curves represent 1:10 serial dilutions of DNA from $100 \mathrm{pg}$ to $10 \mathrm{fg}$ used in the RPA amplification.

RPA: Recombinase polymerase amplification.

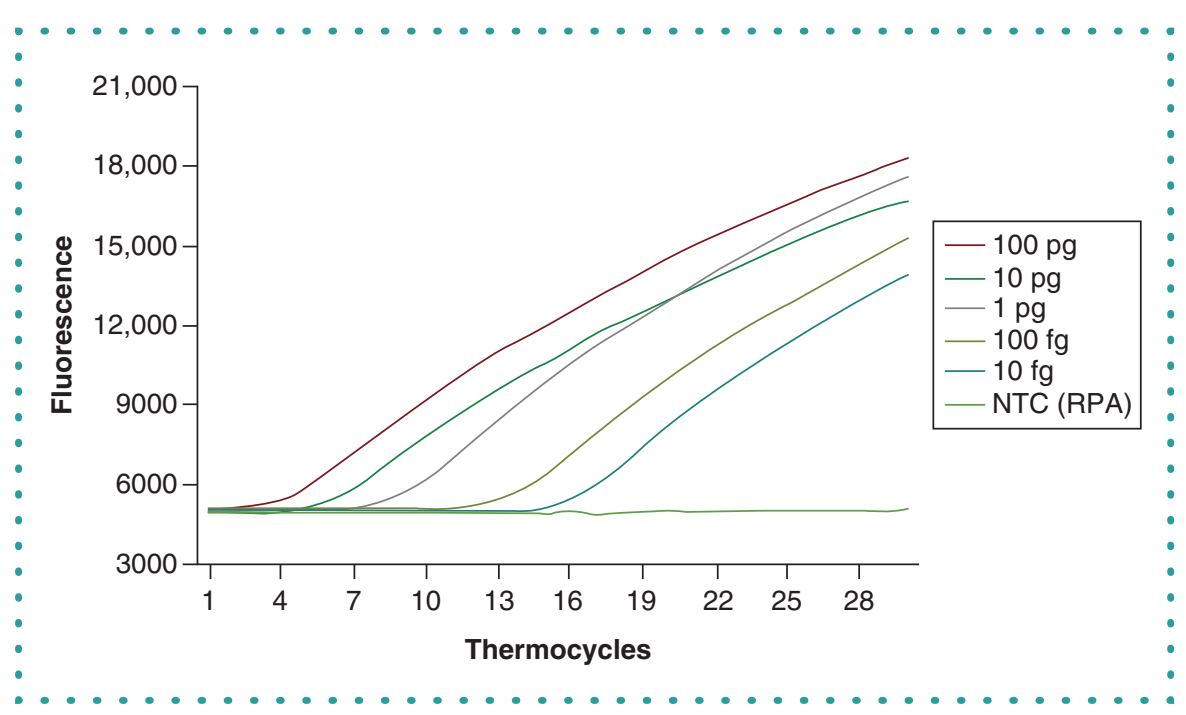

Figure 3. Standard curve of recombinase polymerase amplification-PCR couple when template was Phytophthora cactorum DNA and the coupled PCR is $P$. cactorum specific TaqMan PCR assay. The curves represent 1:10 serial dilutions of DNA from $100 \mathrm{pg}$ to $10 \mathrm{fg}$ used in the RPA amplification. RPA: Recombinase polymerase amplification.

coupled with P. cactorum specific TaqMan PCR assay. The odd shape of the curves in Figure 3 is reflective of the TaqMan assay rather than the RPA assay. Overall, the RPA-PCR couple standard curves were a bit less steep and unevenly spaced compared with relevant PCR assays. The slight decrease in RPA-PCR couple curves steepness possibly indicates inhibition of PCR by RPA reagents. Moreover, as RPA is run first in RPA-PCR couple, the standard curves are more representative of the RPA and therefore unevenly spaced.

In laboratory specificity analysis of RPA-PCR couple, RPA amplification was done with DNA from nontarget taxa and later coupled with the four real-time PCR and one conventional PCR assays. We observed that only the RPA reaction with $P$. cactorum DNA was detected with the coupled $P$. cactorum specific real-time TaqMan and SYBR Green PCR assays. Similarly, only the RPA reaction with $P$. fragariae was detected with the coupled $P$. fragariae specific real-time TaqMan PCR assay, but the coupled $P$. fragariae real-time SYBR Green also detected $P$. cambivora besides $P$. fragariae as expected. Coupling the RPA reactions with genus-specific conventional PCR amplified all 

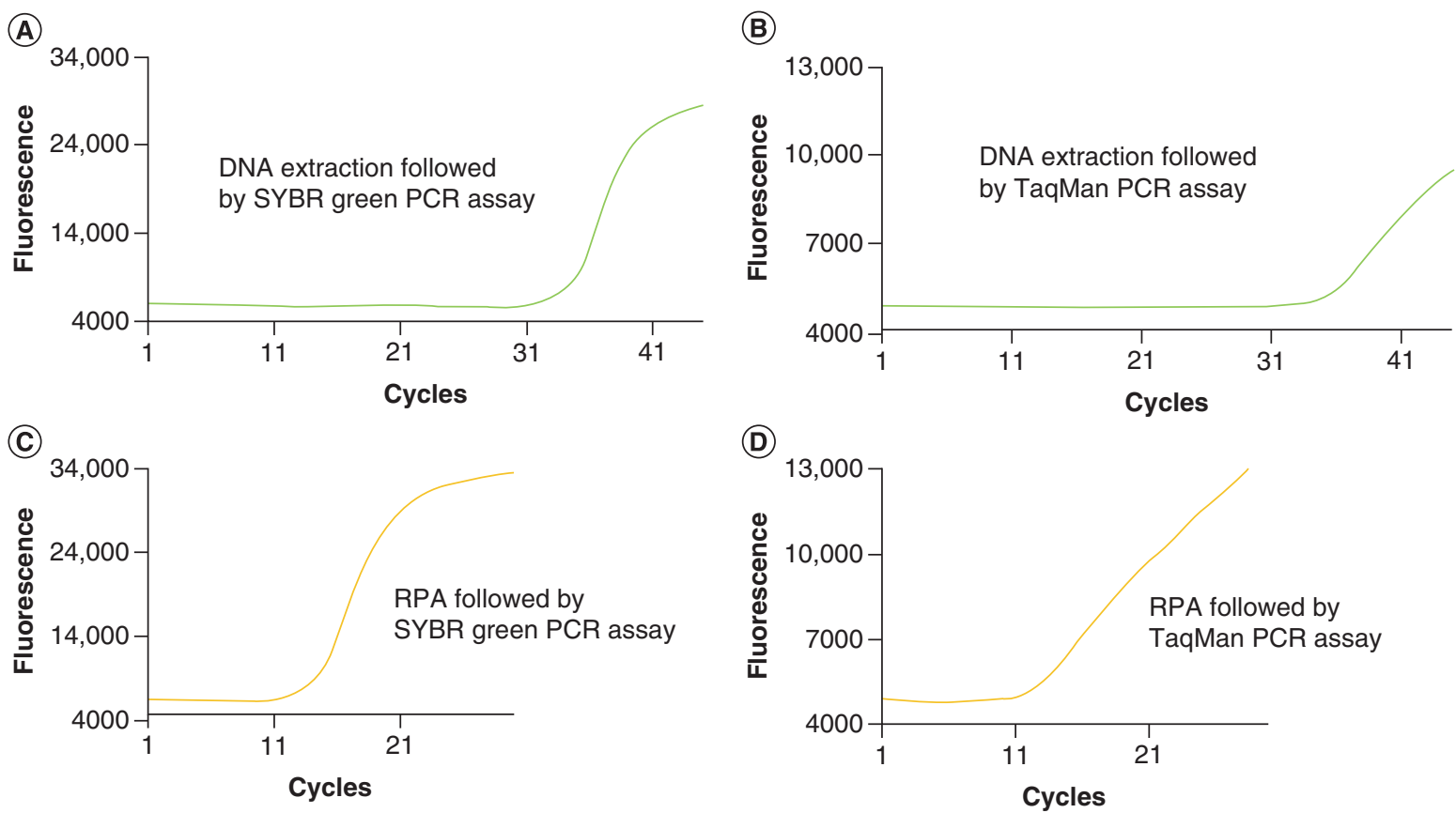

Figure 4. Results of the field validation for a plant crown infected with Phytophthora cactorum. (A \& B) Amplification curves for DNA extraction followed by Phytophthora cactorum specific SYBR Green and TaqMan PCR assay, respectively. (C \& D) Amplification curves for RPA followed by P. cactorum specific SYBR Green and TaqMan PCR assay, respectively.

RPA: Recombinase polymerase amplification.

of the Phytophthora species with no amplification observed with other taxa. Sequencing the Phytophthora amplicons confirmed their species identification.

In laboratory specificity analysis of RPA-PCR couple, DNA of the different taxa was diluted to a range between 50 and $100 \mathrm{pg} / \mu$. Addition of DNA more than $100 \mathrm{pg}$ in an RPA reaction tends to give false amplification in the coupled PCR assays, especially SYBR Green assays, but any false-positive amplifications in SYBR Green PCR assay can be identified through melt curve analysis. Moreover, such high DNA concentrations are not generally present in plant crude macerates.

In RPA-PCR couple optimization, we first screened RPA primers at normal RPA reaction kinetics, then optimized PCR assays and finally tested the coupling of slower kinetics RPA with PCR (RPA-PCR couple). We screened RPA primers at normal reaction kinetics and with a high concentration of template to have better results to compare through gel electrophoresis. We suggest that one can also adopt a reverse approach for optimizing RPA-PCR couple, meaning that first optimizing a PCR assay and later utilizing the PCR assay to compare the slower reaction kinetics RPA reactions added with a different combination of RPA primers. Screening RPA primers through the coupled real-time PCR will exclude the need for RPA reaction clean-up/gel electrophoresis and will provide better data to compare.

\section{Field validation}

For field validation, we compared the RPA-PCR couple method with the method of DNA extraction followed by PCR. We observed some difference in the results of the two methods for the field samples, but all five versions of PCR generated consistent results within each method. The five PCR assays were $P$. fragariae specific TaqMan PCR, $P$. fragariae specific SYBR Green PCR, $P$. cactorum specific TaqMan PCR, P. cactorum specific SYBR Green PCR and Phytophthora genus-specific conventional PCR (Sanger sequencing).

In the process of validation and comparison, we first did RPA-PCR couple from macerates and later extracted DNA from the macerates to perform PCR assays. All DNA extracts that gave no amplification with the five target PCR assays were further tested with a control PCR to exclude the possibility of inhibitors or extraction failure. For all of those samples, the control PCR generated a cycle threshold (Ct) value between 22 and 28, except one crown DNA that had a Ct approximately 33 . The crown sample with the high $\mathrm{Ct}$ was excluded from the validation process. Regarding PCR from DNA extraction, all crown DNA samples amplified with 0.5 and $1 \mu \mathrm{l}$ volume, whereas rootlet DNA samples that had more inhibitors and volumes of 0.25 and $0.5 \mu l$ were mostly successful.

In the comparison of RPA-PCR couple with the method of DNA extraction followed by PCR, the latter produced more positives results. Through RPA-PCR couple, among the 22 rootlet samples investigated, nine were found to be infected with $P$. fragariae, four with $P$. cactorum and one coinfected with both $P$. fragariae and $P$. cactorum. The co-infection was detectable through species-specific PCR assays, but Sanger sequencing was not successful for that sample. Similarly, analysis of 21 crown macerates with RPA-PCR couple 
revealed that eight were infected with $P$. cactorum, whereas 13 crown macerates gave no amplification. Regarding results of PCR from DNA extractions, all positives samples from RPA-PCR couple were also found to be positive. PCR assays from DNA extractions detected three additional weak or late positive samples: one rootlet sample infected with $P$. cactorum and two crown samples contaminated with $P$. fragariae. The Sanger sequencing of the coinfected rootlet samples did not fail but rather generated good reads from the $P$. fragariae atp 9 nad9 marker (possibly due to bias of PCR). All of the Sanger sequences of $P$. fragariae were identical to JF771842 (GenBank accession number), and all sequences of $P$. cactorum were identical to MH094138 (GenBank accession number). In Figure 4, we present results for the method DNA extraction followed by PCR versus RPA-PCR couple for a field plant crown infected with $P$. cactorum.

\section{Conceptualization of RPA-PCR couple}

In prior conceptualization of RPA-PCR couple, we were only aiming at a direct and DNA-extraction-free amplification of the Phytophthora atp9-nad9 marker from infected plant tissue through RPA to proceed with Sanger sequencing, but we faced problems. To succeed with Sanger sequencing, we needed to gel cut the target band, and often the concentration of the target amplicon was not enough. Therefore, we switched to coupling the RPA with PCR. The concept of coupling RPA with PCR was earlier presented by Miles et al. [5], where standard RPA reaction (normal reaction kinetics) was coupled with PCR to sequence and validate some RPA results. We observed that coupling of RPA reactions with PCR for our long target of approximately 500 bp length was not successful for the low target concentrations. Moreover, excessive amplification in RPA reaction, either target or nonspecific, required dilution of the RPA reaction before PCR. Following TwistDx recommendations for long amplicons, we observed that lowering magnesium acetate and primers favored amplification of the long target to some extent, but the issue was resolved through the liquid RPA basic kit (TwistAmp Liquid Basic), which provides flexibility to adjust RPA reaction components. We slowed RPA reaction kinetics by lowering concentrations of primers, MgOAc, ATPs (10X Basic E-Mix), building blocks (dNTPs) and related enzymes (20X Core Reaction Mix), then coupled the slower reaction kinetics with PCR (RPAPCR couple).

The TwistAmp Liquid Basic kit is provided with components of $2 \times$ reaction buffer, $10 \times$ Basic E-Mix, 20× Core Reaction Mix and $280 \mathrm{mM}$ MgOAc. The $2 \times$ reaction buffer is composed of high salt buffer, Tris, potassium acetate, $\mathrm{PEG}$ and $\mathrm{dH}_{2} \mathrm{O}$. Similarly, the $10 \times$ Basic E-Mix contains phosphocreatine, ATP, creatine kinase, high salt buffer and $\mathrm{dH}_{2} \mathrm{O}$. The 20X Core Reaction Mix is composed of GP32, UvsX, UvsY and polymerase (personal communication with TwistDx Inc.).

\section{Advantages of RPA-PCR couple}

RPA has the ability to amplify target nucleic acid directly from simple plant macerates and is a good choice for real-time detection (TwistAmp exo kit) of the pathogen or target region provided that the target marker is short and adequately unique. However, standard RPA cannot efficiently amplify long targets such as 500 bp with adequate sensitivity, and long amplicons can be desirable in different applications such as Sanger sequencing. Similarly, we observed that some nonspecific by-products generated in RPA were not removed through routine PCR purification kits, and purification of amplicons required gel extraction. RPA assays have also been reported tolerating up to nine mismatches. In contrast to RPA, the traditional method of plant DNA extraction followed by PCR sometimes encounters the problem of amplification inhibitors. The inhibitors enter the DNA sample during the lengthy process of DNA extraction, and therefore we proposed excluding the DNA extraction step and replacing it with RPA. An RPA run at slower reaction kinetics can amplify a long target in limited quantity. The limited or low number of amplicons is not a problem, as a coupled PCR can exponentially amplify those limited amplicons. We observed that slowing the RPA reaction not only favored amplification of long targets but also excluded the need for diluting the RPA reaction prior to doing PCR and provided a better resolution for quantification through real-time quantitative PCR assays compared with standard RPA assays (TwistAmp exo kit). Moreover, through RPA-PCR couple, we experienced quicker amplification of our target (compared with traditional methods) and simple purification of amplicons (compared with standard RPA) through a routine PCR purification kit. In short, in RPA-PCR couple, the RPA takes the role of initial replication of the target from crude macerate, whereas PCR takes responsibility for more specific and exponential amplification of the target.

Although RPA-PCR couple requires additional steps for preparation of RPA reaction mix, TwistDx Inc. also provides a custom freezedrying service for RPA reaction pellets with variation in the concentration of components. Moreover, PCR in RPA-PCR couple also runs for fewer cycles (lesser time). The RPA-PCR couple procedure for a batch of 10-20 samples takes 5-7 h, whereas results for the traditional method of plant DNA extraction followed by real-time PCR are usually available the next day (8-11 $\mathrm{h}$ in total). It is notable that we utilized the cost-effective but lengthy DNA extraction method of Till et al. [24]; however, commercial kits such as the DNeasy Plant Mini kit (Qiagen, Germany) have relatively shorter procedures. Regarding the cost of RPA-PCR couple, it is comparable with the traditional method. For instance, the current price of a TwistAmp Liquid Basic ( 100 reactions) is EUR200, whereas the DNeasy Plant Mini kit (50 columns) is priced at EUR207. In Table 2, we compare the performance of RPA-PCR couple with that of the traditional method of DNA extraction followed by PCR.

\section{Applications of RPA-PCR couple}

We conceived the idea of RPA-PCR couple for plant diagnostics, but it may prove beneficial for other difficult samples encountering the PCR inhibitors problem when DNA is extracted, such as food or fecal samples [27]. It is notable that woody plant tissues have a high content of phenolic compounds and polysaccharides that are co-extracted along with nucleic acid extraction and result in PCR 


\begin{tabular}{|c|c|c|c|c|}
\hline Method & Sensitivity & Specificity & Time duration & Cost \\
\hline DNA extraction followed by PCR & $\begin{array}{l}\text { PCR consistently detected down to } 1 \mathrm{fg} \\
\text { Phytophthora genomic DNA }\end{array}$ & Satisfactory & $5-7 \mathrm{~h}$ & Comparable \\
\hline RPA-PCR couple & $\begin{array}{l}\text { RPA-PCR couple consistently detected down to } \\
10 \text { fg Phytophthora genomic DNA }\end{array}$ & Equally satisfactory & $8-11 \mathrm{~h}$ & Comparable \\
\hline
\end{tabular}

inhibition [28], but RPA has shown success in amplifying targets from woody plant macerates. The RPA assay of Miles et al. [5] was able to amplify the target from Umbellularia californica crude macerate. Similarly, the RPA assay of Zhang et al. [7] also amplified the target from some Prunus plants. Therefore, we expect the RPA-PCR couple concept to work with different recalcitrant sample macerates. We have presented the method for amplification of pathogen DNA from plant macerates, but it is equally applicable to amplify plant or macerate DNA. Moreover, RPA-PCR couple should also be able to amplify RNA targets by adding suitable reverse transcriptase in an RPA reaction.

Applying the RPA-PCR couple method to identification markers and doing Sanger sequencing afterward can expedite species identification. In the current study, utilization of the unique intergenic atp9-nad9 marker provided a rapid Phytophthora identification assay with the potential to identify almost all Phytophthora species $[5,22]$. The assay is about crudely macerating Phytophthora-infected plant tissue in water and transferring the macerate supernatant into the RPA reaction added with forward primer 'ATP9F-M9' and reverse primer 'Phy_Gen_R20.' The RPA reaction is transferred to conventional PCR assay ('Phy_Gen_SeqF4' and 'Phy_Gen_SeqR8' primers), and the PCR product is cleaned and Sanger sequenced. It is notable that we have only tested the assay with ten Phytophthora species due to limited resources. Moreover, the assay will not be applicable to Phytophthora bisheria or Phytophthora frigida due to the possible unusual gene order of atp9 and nad 9 genes [22].

RPA-PCR couple should be equally applicable to long and short targets, and it may even work with a target longer than $500 \mathrm{bp}$. In RPA-PCR couple optimization, we first screened RPA primers and found the optimum primer pair amplifying a target of approximately 500 bp length. Next, we tried to design a conventional PCR with a subtarget length of approximately $450 \mathrm{bp}$, but we ended up with a subtarget length of approximately 300 bp due to difficulty in primer designing from the GC-poor atp-nad 9 marker.

\section{Preventing contamination}

Although RPA generates a limited product in RPA-PCR couple, opening RPA tubes to transfer reactions into PCR poses risk of contamination. The RPA strip opening and reaction transfer should be carefully accomplished in some suitable hood of the post-PCR laboratory. Similarly, filter tips or positive displacement tips should be utilized to avoid contamination. Good molecular laboratory practice of physically separating pre- and postamplification laboratories, preparing macerates and reactions reagents in the preamplification laboratory, and including no template control (NTC) guarantee quality of results. The RPA reaction should also be added with an NTC (RPA NTC), which can be further coupled with PCR (coupled NTC). PCR should also include its own NTC. In addition, it is important to mark RPA lid strips to avoid direction inversion of the lid strip. In RPA-PCR couple, either RPA or PCR can be provided with the UDG enzyme and the dTTP partially or completed replaced with dUTP. Importantly, the UDG system cannot be applied to both RPA and PCR in the couple, as the product of RPA is being utilized by the coupled PCR as the initial template.

\section{Future perspective}

Isothermal nucleic acid amplification technologies including RPA often suffer from background amplification problems, and their utilization for initial and limited amplification of target nucleic acid to replace the DNA extraction step provides new insight. In this proof-ofconcept study, we demonstrated RPA-PCR couple as a time-saving procedure to amplify target DNA from simple macerates, excluding the need for laborious plant DNA extraction. Although we utilized the Phytophthora identification marker atp-nad 9 and strawberry Phytophthora pathogens to prove the functioning of our RPA-PCR couple concept, the concept is quite general. We expect that this method can also amplify nucleic acid targets from other recalcitrant samples. To apply the concept to some defined targets and available PCR assays, one would only need to design RPA primers from flanking regions of their targets and screen them to find an optimal pair.

\section{Supplementary data}

Supplementary Table 1 provides a list of the taxa utilized in laboratory evaluations of specificity and their isolate numbers, sources and origins of recovery. To view the supplementary data please visit the journal website at: www.future-science.com/doi/suppl/10.2144/btn2020-0065 


\section{Author contributions}

MA Munawar completed most of the laboratory work and wrote the manuscript, whereas all other coauthors contributed to improving the manuscript. F Martin also contributed by conceptualizing and improving experiment designs.

\section{Acknowledgments}

The authors thank the technical support team of TwistDx Inc. for introducing us to TwistAmp Liquid Basic and generously answering our questions.

\section{Financial \& competing interests disclosure}

Initial optimization of RPA-PCR couple was accomplished during the 'Tauti voi ei!' project funded by Euroopan maaseudun kehittämisen maatalousrahasto (European Agricultural Fund for Rural Development/EAFRD), Pohjois-Savon ELY-Keskus. MA Munawar also received personal grants from the Finnish Cultural Foundation and Olvi Foundation, Finland, to finalize the manuscript. The authors have no other relevant affiliations or financial involvement with any organization or entity with a financial interest in or financial conflict with the subject matter or materials discussed in the manuscript apart from those disclosed.

No writing assistance was utilized in the production of this manuscript.

\section{Open access}

This work is licensed under the Attribution-NonCommercial-NoDerivatives 4.0 Unported License. To view a copy of this license, visit http: //creativecommons.org/licenses/by-nc-nd/4.0/

\section{Protocols.io link}

https://www.protocols.io/view/protocol-for-rpa-pcr-couple-bifrkbm6

\section{References}

Papers of special note have been highlighted as: $\bullet$ of interest; $\bullet \bullet$ of considerable interest

1. Martin FN, Abad ZG, Balci Y, Ivors K. Identification and detection of Phytophthora: reviewing our progress, identifying our needs. Plant Dis. 96(8), 1080-1103 (2012).

2. Piepenburg O, Williams $\mathrm{CH}$, Stemple DL, Armes NA. DNA detection using recombination proteins. PLoS Biol. 4(7), e204 (2006).

3. Li J, Macdonald J, von Stetten F. Review: a comprehensive summary of a decade development of the recombinase polymerase amplification. Analyst 144(1), 31-67 (2019).

.. Summarizes the ability of recombinase polymerase amplification (RPA) to tolerate inhibitors and its tendency toward nonspecific detection of close targets.

4. Kumar PV, Sharma SK, Rishi N, Ghosh DK, Baranwal VK. An isothermal based recombinase polymerase amplification assay for rapid, sensitive and robust indexing of citrus yellow mosaic virus. Acta Virol. 62(1), 104-108 (2018).

5. Miles TD, Martin FN, Coffey MD. Development of rapid isothermal amplification assays for detection of Phytophthora spp. in plant tissue. Phytopathology 105(2), 265-278 (2015).

.• Coupled RPA and PCR, and it also describes crude maceration and the atp9-nad9 marker. The RPA assay designed worked with woody plant tissue macerate.

6. Li R, Fuchs MF, Perry KL, Mekuria T, Zhang S. Development of a fast Amplifyrp Acceler8 diagnostic assay for grapevine red blotch virus. J. Plant Pathol. 99(3), 657-662 (2017).

7. Zhang S, Ravelonandro M, Russell $P$ et al. Rapid diagnostic detection of plum pox virus in Prunus plants by isothermal AmplifyRP using reverse transcription-recombinase polymerase amplification. J. Virol. Methods 207, 114-120 (2014).

- The RPA assay designed worked with woody plant tissue macerate.

8. Kalyebi A, Aisu G, Ramathani I, Ogwang J, McOwen N, Russell P. Detection and identification of etiological agents (Liberibacter spp.) associated with citrus greening disease in Uganda. Uganda J. Agric. Sci. 16(1), 43-54 (2015).

9. Ghosh DK, Kokane SB, Kokane AD et al. Development of a recombinase polymerase based isothermal amplification combined with lateral flow assay (HLB-RPA-LFA) for rapid detection of "Candidatus Liberibacter asiaticus.". PLoS ONE 13(12), e0208530 (2018).

10. Karakkat BB, Hockemeyer K, Franchett M, Olson M, Mullenberg C, Koch PL. Detection of root-infecting fungi on cool-season turfgrasses using loop-mediated isothermal amplification and recombinase polymerase amplification. J. Microbiol. Methods 151, 90-98 (2018).

11. Qian W, Lu Y, Meng Y et al. Field detection of citrus Huanglongbing associated with 'Candidatus Liberibacter Asiaticus' by recombinase polymerase amplification within 15 min. J. Agric. Food Chem. 66(22), 5473-5480 (2018).

12. Ahmed FA, Larrea-Sarmiento A, Alvarez AM, Arif M. Genome-informed diagnostics for specific and rapid detection of Pectobacterium species using recombinase polymerase amplification coupled with a lateral flow device. Sci. Rep. 8(1), 1-11 (2018)

13. Si Ammour M, Bilodeau GJ, Tremblay DM et al. Development of real-time isothermal amplification assays for on-site detection of Phytophthora infestans in potato leaves. Plant Dis. 101(7), 1269-1277 (2017).

14. Silva G, Oyekanmi J, Nkere CK, Bömer M, Kumar PL, Seal SE. Rapid detection of potyviruses from crude plant extracts. Anal. Biochem. 546, 17-22 (2018).

15. Wambua L, Schneider B, Okwaro A et al. Development of field-applicable tests for rapid and sensitive detection of Candidatus Phytoplasma oryzae. Mol. Cell. Probes 35, 44-56 (2017).

16. Patel P, Abd El Wahed A, Faye $O$ et al. A field-deployable reverse transcription recombinase polymerase amplification assay for rapid detection of the chikungunya virus. PLoS Neglected Trop. Dis. 10(9), e0004953 (2016).

17. Yang Y, Qin X, Sun Y, Cong G, Li Y, Zhang Z. Development of isothermal recombinase polymerase amplification assay for rapid detection of porcine circovirus type 2. BioMed Res. Int. 2017, 8403642 (2017).

18. Moore MD, Jaykus LA. Development of a recombinase polymerase amplification assay for detection of epidemic human noroviruses. Sci. Rep. 7, 40244 (2017).

19. Abd El Wahed A, El-Deeb A, El-Tholoth $\mathrm{M}$ et al. A portable reverse transcription recombinase polymerase amplification assay for rapid detection of foot-and-mouth disease virus. PLoS ONE 8(8), e71642 (2013).

20. Daher RK, Stewart G, Boissinot M, Boudreau DK, Bergeron MG. Influence of sequence mismatches on the specificity of recombinase polymerase amplification technology. Mol. Cell. Probes 29(2), 116-121 (2015).

-. Highlights the tendency of RPA to tolerate mismatches (nonspecificity).

21. Boyle DS, Lehman DA, Lillis L et al. Rapid detection of HIV-1 proviral DNA for early infant diagnosis using recombinase polymerase amplification. mBio 4(2), e00135-13 (2013).

22. Bilodeau GJ, Martin FN, Coffey MD, Blomquist CL. Development of a multiplex assay for genus- and species-specific detection of Phytophthora based on differences in mitochondrial gene order. Phytopathology 104(7), 733-748 (2014).

- Describes development of the Phytophthora identification marker atp9-nad9.

23. Munawar M, Toljamo A, Martin F, Kokko H. Recombinase Polymerase Amplification Assay for fast, sensitive and on-site detection of Phytophthora cactorum without DNA extraction. Eur. J. Hortic. Sci. 84(1), 14-19 (2019).

- Describes maceration of plant tissue with an Eppendorf tube and a plastic pestle. 
24. Till BJ, Jankowicz-Cieslak J, Huynh OA, Beshir MM, Laport RG, Hofinger BJ. Low-Cost Methods for Molecular Characterization of Mutant Plants: Tissue Desiccation, DNA Extraction and Mutation Discovery: Protocols. Springer, Cham, Switzerland (2015).

25. Tooley PW, Martin FN, Carras MM, Frederick RD. Real-time fluorescent polymerase chain reaction detection of Phytophthora ramorum and Phytophthora pseudosyringae using mitochondrial gene regions. Phytopathology 96(4), 336-345 (2006).

26. Dwight Z, Palais R, Wittwer CT. uMELT: prediction of high-resolution melting curves and dynamic melting profiles of PCR products in a rich web application. Bioinformatics $27(7)$, 1019-1020 (2011).

27. Schrader C, Schielke A, Ellerbroek L, Johne R. PCR inhibitors-occurrence, properties and removal. J. Appl. Microbiol. 113(5), 1014-1026 (2012).

28. Osman F, Rowhani A. Application of a spotting sample preparation technique for the detection of pathogens in woody plants by RT-PCR and real-time PCR (TaqMan). J. Virol. Methods 133(2), 130-136 (2006). 
